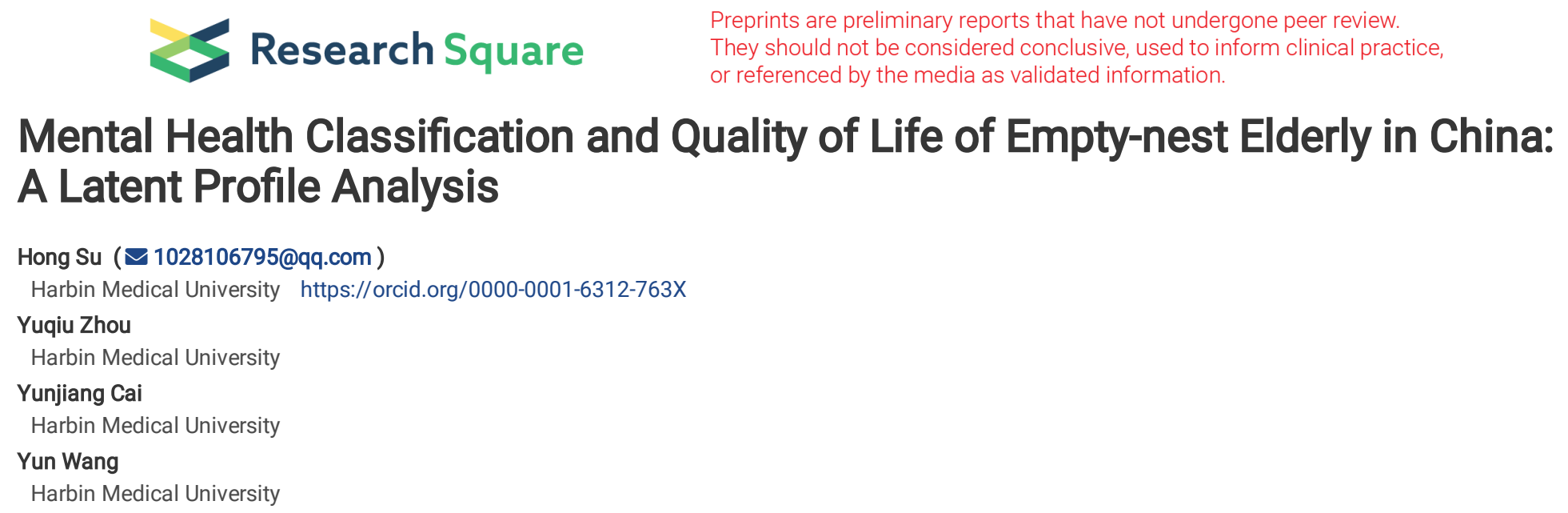

\section{Mental Health Classification and Quality of Life of Empty-nest Elderly in China: A Latent Profile Analysis}

\title{
Research
}

Keywords: Mental health, Quality of life, Empty-nest elderly, Latent profile analysis

Posted Date: July 7th, 2021

DOI: https://doi.org/10.21203/rs.3.rs-658711/v1

License: (9) (1) This work is licensed under a Creative Commons Attribution 4.0 International License. Read Full License 


\section{Abstract}

Background冈Latent profile analysis was used to identify the profiles of empty-nest elderly's mental health. Associations of the profiles with quality of life were then examined.

Methods: Three hundred and fifty empty-nest elderly adults in China were investigated with Elderly Mental Health Questionnaire and the Chinese version of the SF-36. Latent profile analysis was used to analyze the data.

Results: A three-profile mental health model provided the best fit to the data. The resulting profiles were low mental health, moderate mental health, and high mental health. There were significant differences in quality of life among different profiles of mental health.

Conclusion: The results provide a new and expanded view of empty nesters' mental health, which may be used to improve empty-nest elderly's quality of life.

\section{Background}

China has the world's largest elderly population. The China Statistical Yearbook reported that adults aged 60 and above accounted for $17.9 \%$ of the total population by the end of 2018[1]. Empty-nest elderly are a special group among the elderly, which refers to older adults who have no children or whose children have already left home and thus live alone or with their spouse or older parents[2]. According to statistics, the number of empty-nest elderly in China has reached 120 million by the end of 2017 , accounting for about $51.3 \%$ of the total number of elderly people[3]. It was estimated that the proportion of families with empty-nest elderly will reach $90 \%$ of all families in China by $2030[4]$. Due to the lack of care from their children, the mental health status of the empty-nest elderly is worse than that of the ordinary elderly[5], empty-nest elderly are more likely to suffer from physical, psychological, and social problems[6]. Poor mental health status reduces the quality of life of the empty-nest elderly, damages their social function, and leads to the decline of cognitive function, life satisfaction and well-being[7]. A great deal of theoretical and empirical work completed over the past few decades has substantially increased our understanding of the conditions and factors influencing mental health of empty-nest elderly.

Scholarly interest in mental health among empty-nest elderly continues to grow, yet most research has adopted a variable-centered approach which focuses on testing the relationships of each facet of mental health with other variables[8]. Although such an approach provides valuable information about the direct and unique association of each facet of mental health with other variables, it ignores the possibility that (a) distinct combinations of mental health profiles exist in the population and (b) these mental health profiles may correspond to differences in other variables. This perspective is consistent with taking a personcentered approach to conceptualizing mental health, which recognizes that distinct mental health profiles might exist. Investigation of these profiles through the person-centered approach might reveal unique insights into the effects of mental health profiles on other variables.

Data-driven techniques are a fruitful way of studying the psychosocial patterning evident during this life stage. Specifically, latent profile analysis (LPA) performs this process in a more rigorous statistical manner. LPA is a person-centered approach that classifies samples into more meaningful subgroups than variable-centered approaches, handles interaction effects, and is not affected by outliers[9]. Thus, this probability-based and individual-centered approach could yield lower misclassification and missing rates for participants[10]. LPA also facilitates the discovery of heterogeneous subpopulations with similar attributes across multiple dimensions of interest[11]. Use of a person-centered approach enables identification of how different subpopulations of empty-nest elderly characteristically use distinct combinations of different mental health to affect their quality of life.

Healthy aging is manifested in not only the extension of the life of the older people but also, more importantly, the improvement of their quality of life[12]. However, the overall health condition of older adults is not optimistic. Studies have showed that with the growth of age, the physical health, self-care ability and cognitive function of the older adults tend to decline[13]. As a vulnerable group, shrinking social resources and a lack of emotional support for empty nesters could lead to a greater risk of health and psychological problems[14]. According to the psychological stress theory, physical health is closely related to psychological factors. Long-term bad mood will lead to the decline of human immunity, affect people's physical function and reduce the quality of life[15]

Quality of life(QoL) refers to a person's personal perception of psychological, emotional, and social status[16]. As a special group of the elderly, the empty-nest elderly lacked family care for a long time, their emotional comfort was scarce and their ability to use all kinds of social basic resources independently was weak, so the QoL of them needs to be paid more attention. Mental health plays an important role in maintaining the quality of life of empty-nest elderly [17]. However, previous research has been limited as it has not examined the relationship between empty-nest elderly's mental health profiles and their quality of life.

The aim of this study was to investigate whether different mental health profiles exist among Chinese empty-nest elderly. LPA was used to classify empty-nest elderly who showed similar mental health patterns into a certain profile. Associations of the profiles with quality of life were then examined.

\section{Methods}

\section{Ethics statement}

The study protocol was in accordance with the ethical standards and was approved by the Ethics Committee of Harbin Medical University. Written informed consent was obtained from each participant. Information collected from all participants was kept confidential and anonymous.

\section{Study design and participants}

A multistage stratified random sampling method was used. Step 1:We selected one region randomly from three regions of Chifeng City, and then six streets were selected from this region randomly. Finally, we selected three communities from each street randomly with a total of 18 residential committees extracted. 
Step 2: Using simple random sampling in accordance with the information provided by community workers, empty-nest elderly were selected randomly from the 18 residential committees. All the respondents completed a household questionnaire survey and provided informed consent after receiving information about the goals and the methods of the investigation. Initially, 398 questionnaires were issued and 350 were received, for a recovery rate of $87.94 \%$. Participants were e measured between August 2016 to March 2018.

The inclusion criteria included: (1) local residents with age $\geqq 60$; (2) understand the survey content and agree to participate; (3) living alone or with their spouse or older parents. Exclusion criteria: (1)childless ;(2)severe cognitive impairment or mental illness.

\section{Measures}

\section{Demographic characteristics}

The demographic characteristics were collected with a general information questionnaire designed by our panel, which included gender, age, education, marital status, household monthly income.

\section{Measurement of Mental Health}

The Elderly Mental Health Questionnaire, used to assess mental health status in the elderly, was developed by Zhenyun Wu[18]. Mental health was measured using five dimensions, which included ego, emotion, adaptive capacity, interpersonal communication, and cognitive function. The questionnaire consists of 68 items. Each item has four response categories ranging from "do not agree" (1 point) to "agree" (4 points), and higher scores indicated better mental health. The questionnaire has been used in Chinese older men and demonstrated good reliability and validity, with Cronbach's alphas for the total scale and subscales of 0.58-0.89.

\section{Measurement of QoL}

This study applied the Chinese version of the SF-36, which consists of 36 items to evaluate the quality of life (QOL) of participants[19]. One item assesses the perceived change in health status, and the remaining 35 items assess the physical and mental components of health[20]. The SF-36 included 8 dimensions: physical functioning (PF), role-physical (RP); bodily pain (BP); general health (GH); vitality (VT); social functioning(SF); role-emotional (RE) and mental health $(\mathrm{MH})$. These 8 dimensions are summarized in 2 categories that physical component summary (PCS) and mental component summary (MCS). PCS includes PF, RP, BP and GH. MCS includes VT, SF, RE, and MH. Each dimension score was converted to a range from 0 to 100 , with a high score indicating better levels of functioning. This scale has good reliability in the evaluation of the quality of life of the elderly,the Cronbach's a of the SF-36 was $0.780[21]$.

\section{Data analyses}

Means and standard deviations for all variables were computed with SPSS software version 22.0, Spearman correlations were used to analyse the correlation between age, gender, education level, marital status, household monthly income, mental health, and quality of life, P-values equal or lower than 0.05 were considered statistically significant.

The latent profile approach (LPA) was conducted in Mplus 7.4 in order to explore the potential classification of mental health among empty-nest older adults. The best-fitting model was selected by examining indices of model fit and interpretability of the model. To determine the model fit, Akaike information criterion (AIC), Bayesian information criterion (BIC), and adjusted BIC (aBIC), Lo-Mendell-Rubin (LMR), Bootstrapped Likelihood Ratio Test (BLRT), and Entropy were adopted and examined. The AIC provided a measure of fit considering the number of model parameters. The BIC and aBIC are indicators in which both sample size and number of model parameters are considered. Smaller values of AIC, BIC, aBIC suggest an improved model fit. Entropy is a means to evaluate how well each group originating from LPA, with values equal or greater than 0.80 demonstrating a better result[22]. LMR and BLRT are adopted to compare the estimated model and a model with k-1 profile(s), with k indicating the number of profiles (Nylund et al.,2007). For the LMR and BLRT, a lower and significant pvalue signifies a superior estimated model compared to the model with one less profile[23].

To examine the effect of age, gender, degree of education, marital status and household monthly income on mental health profiles, a three-step approach was adopted[24] based on a kind of regression mixture modeling with Mplus, including covariates[25]. Data analyses were performed in Mplus 7.4.

\section{Results}

\section{Description of the participants}

A total of 350 participants with a mean age of 71.05 years $(S D=6.22)$ completed the survey. The percentage of participants with an education level from primary school to university was $51.1 \%-8 \%$. Of all the participants, $72.9 \%$ reported to be married. 154 (44\%) were male, and $196(56 \%)$ were female as shown in Table 1.

Table 1 Demographic characteristics. 


\begin{tabular}{|c|c|c|c|c|}
\hline Demographics & Classification & Frequency & Percent & Cumulative percent \\
\hline \multirow[t]{2}{*}{ Gender } & Female & 196 & 56 & 56 \\
\hline & Male & 154 & 44 & 100 \\
\hline \multirow[t]{3}{*}{ Age } & $60-70$ & 155 & 44.3 & 44.3 \\
\hline & $70-80$ & 143 & 40.9 & 85.1 \\
\hline & $\triangle 80$ & 52 & 14.9 & 100 \\
\hline \multirow[t]{4}{*}{ Education level } & Primary school and below & 179 & 51.1 & 51.1 \\
\hline & Secondary school & 93 & 26.6 & 77.7 \\
\hline & High school & 50 & 14.3 & 92.0 \\
\hline & College graduate or above & 28 & 8.0 & 100 \\
\hline \multirow[t]{2}{*}{ Marital status } & Married & 255 & 72.9 & 72.9 \\
\hline & $\begin{array}{l}\text { Single (never married, divorced, } \\
\text { separated and widowed) }\end{array}$ & 95 & 17.1 & 100 \\
\hline \multirow[t]{3}{*}{ Household monthly income } & Low $(\leq 1000 \mathrm{rmb})$ & 176 & 50.3 & 50.3 \\
\hline & Middle (1001-2000 rmb) & 127 & 36.3 & 86.6 \\
\hline & High (>2000 rmb) & 47 & 13.4 & 100 \\
\hline
\end{tabular}

\section{Correlation analyses between the variables}

As presented in Table 2, total score of mental health of the elderly was positively related with education level, household monthly income, role-physical, pysical functioning, general health, vitality, social functioning, role-emotional, mental health, total score of quality of life $\left(r=0.618^{\star \star}, 0.407^{\star \star}, 0.711^{\star *}, 0.869^{\star \star}, 0.681^{\star *}\right.$, $0.554^{\star *}, 0.470^{\star *}, 649^{\star *}, 0.677^{\star *}$ and $0.701^{\star *}$, respectively). The total score of mental health of the elderly was negatively correlated with gender, age, marital status, bodily pain $\left(r=-0.108^{*},-0.395^{\star *},-0.131^{\star}\right.$ and $-0.303^{* *}$, respectively $)$.

Table 2 Correlation analysis among variables

\begin{tabular}{|c|c|c|c|c|c|c|c|c|c|c|c|c|c|c|}
\hline & 1 & 2 & 3 & 4 & 5 & 6 & 7 & 8 & 9 & 10 & 11 & 12 & 13 & 14 \\
\hline 1.gender & 1.000 & .002 & $-.169^{\star \star}$ & -.013 & -.062 & $-.108^{\star}$ & .051 & -.056 & .043 & -.029 & $.199^{\star}$ & $.206^{\star *}$ & -.013 & $.129^{\star}$ \\
\hline 2.age & .002 & 1.000 & $-.431^{* *}$ & $-.288^{\star *}$ & .019 & $-.395^{\star *}$ & $-.372^{\star *}$ & $-.298^{\star *}$ & $.144^{\star \star}$ & $-.332^{\star *}$ & $-.276^{\star *}$ & $-.261^{\star *}$ & $-.319^{\star *}$ & $-.336^{\star *}$ \\
\hline 3.Eu & $-.169^{\star *}$ & $-.431^{* *}$ & 1.000 & $.576^{\star \star}$ & -.033 & $.618^{\star *}$ & $.575^{\star \star}$ & $.551^{\star \star}$ & $-.314^{* *}$ & $.562^{\star \star}$ & $.408^{\star *}$ & $.355^{\star *}$ & $.523^{\star *}$ & $.509^{\star *}$ \\
\hline 4. Hmi & -.013 & $-.288^{\star \star}$ & $.576^{* \star}$ & 1.000 & .062 & $.407^{\star \star}$ & $.400^{\star \star}$ & $.409^{\star \star}$ & $-.196^{\star *}$ & $.373^{\star *}$ & $.311^{\star *}$ & $.264^{\star \star}$ & $.343^{\star \star}$ & $.368^{\star \star}$ \\
\hline 5.Mar & -.062 & .019 & -.033 & .062 & 1.000 & $-.131^{*}$ & $-.110^{\star}$ & $-.130^{*}$ & .016 & -.071 & $-.126^{*}$ & -.097 & -.076 & $-.124^{*}$ \\
\hline 6.EMH & $-.108^{\star}$ & $-.395^{\star *}$ & $.618^{\star \star}$ & $.407^{\star \star}$ & $-.131^{*}$ & 1.000 & $.711^{\star \star}$ & $.869^{\star \star}$ & $-.303^{\star *}$ & $.681^{\star *}$ & $.554^{\star \star}$ & $.470^{\star *}$ & $.649^{\star *}$ & $.677^{\star *}$ \\
\hline 7.RP & .051 & $-.372^{\star *}$ & $.575^{\star \star}$ & $.400^{\star \star}$ & $-.110^{\star}$ & $.711^{\star *}$ & 1.000 & $.638^{\star \star}$ & $-.382^{\star *}$ & $.728^{\star *}$ & $.826^{\star *}$ & $.730^{\star *}$ & $.720^{\star *}$ & $.925^{\star *}$ \\
\hline 8.PF & -.056 & $-.298^{* *}$ & $.551^{\star \star}$ & $.409^{\star \star}$ & $-.130^{\star}$ & $.869^{\star *}$ & $.638^{\star \star}$ & 1.000 & $-.328^{\star \star}$ & $.615^{\star \star}$ & $.509^{\star \star}$ & $.417^{\star *}$ & $.584^{\star *}$ & $.608^{\star \star}$ \\
\hline 9.BP & .043 & $.144^{\star *}$ & $-.314^{\star \star}$ & $-.196^{* *}$ & .016 & $-.303^{\star \star}$ & $-.382^{\star *}$ & $-.328^{\star *}$ & 1.000 & $-.341^{\star \star}$ & $-.285^{\star *}$ & $-.257^{\star *}$ & $-.343^{\star \star}$ & $-.325^{\star \star}$ \\
\hline 10.GH & -.029 & $-.332^{* *}$ & $.562^{\star \star}$ & $.373^{\star \star}$ & -.071 & $.681^{* *}$ & $.728^{\star \star}$ & $.615^{\star \star}$ & $-.341^{\star *}$ & 1.000 & $.483^{\star *}$ & $.390^{\star *}$ & $.976^{\star *}$ & $.581^{\star *}$ \\
\hline 11.VT & $.199^{\star *}$ & $-.276^{\star *}$ & $.408^{\star \star}$ & $.311^{\star \star}$ & $-.126^{*}$ & $.554^{\star \star}$ & $.826^{\star \star}$ & $.509^{\star \star}$ & $-.285^{\star *}$ & $.483^{\star *}$ & 1.000 & $.800^{\star *}$ & $.469^{\star *}$ & $.933^{* *}$ \\
\hline 12.SF & $.206^{\star \star}$ & $-.261^{* *}$ & $.355^{* \star}$ & $.264^{\star \star}$ & -.097 & $.470^{\star *}$ & $.730^{\star \star}$ & $.417^{\star \star}$ & $-.257^{\star \star}$ & $.390^{\star \star}$ & $.800^{\star *}$ & 1.000 & $.376^{\star *}$ & $.848^{\star \star}$ \\
\hline 13.RE & -.013 & $-.319^{\star *}$ & $.523^{\star \star}$ & $.343^{\star \star}$ & -.076 & $.649^{\star \star}$ & $.720^{\star \star}$ & $.584^{\star \star}$ & $-.343^{\star \star}$ & $.976^{\star \star}$ & $.469^{\star \star}$ & $.376^{\star \star}$ & 1.000 & $.564^{\star \star}$ \\
\hline 14.MH & $.129^{*}$ & $-.336^{* *}$ & $.509^{\star *}$ & $.368^{\star \star}$ & $-.124^{*}$ & $.677^{\star *}$ & $.925^{\star \star}$ & $.608^{\star \star}$ & $-.325^{\star *}$ & $.581^{\star \star}$ & $.933^{\star *}$ & $.848^{\star *}$ & $.564^{\star *}$ & 1.000 \\
\hline 15.QOL & $.143^{\star *}$ & $-.354^{\star \star}$ & $.499^{* \star}$ & $.355^{\star *}$ & $-.135^{\star}$ & $.701^{\star \star}$ & $.891^{\star *}$ & $.616^{\star *}$ & $-.110^{*}$ & $.687^{\star *}$ & $.886^{\star *}$ & $.817^{\star \star}$ & $.669^{* *}$ & $.935^{\star *}$ \\
\hline
\end{tabular}

Edu: education level; Hmi: Household monthly income;Mar:Marital status; EMH:Elderly Mental Health, PF:Pysical functioning,RP: role-physical; BP:bodily pain; GH:general health;VT: vitality ;SF: social functioning; RE:role-emotional;mental health (MH);QOL:Total score of quality of life 
Table3 provides the fit indices for the LPA models. Two- and three-class models both had significant LMR and BLRT results at $\mathrm{p}<0.05$. However, the threeclass solution provided a better fit than the two-class model, as AIC and BIC for the former were lower, while also showing a good value of Entropy. Therefore, the three-class model was selected as most appropriate.

Table 3 Model fit indices for one- to five-profile pattern of Mental Health dimensions and profile prevalence (\%) of LPA

\begin{tabular}{|llllllllll|}
\hline Profiles & $\mathrm{m}$ & $\log (\mathrm{L})$ & $\mathrm{AIC}$ & $\mathrm{BIC}$ & aBIC & Entropy & LMR & BLRT & Category probability (\%) \\
\hline 1 & 10 & -5452.36 & 10924.72 & 10963.30 & 10931.58 & & & & \\
\hline 2 & 16 & -4500.29 & 9032.58 & 9094.31 & 9043.55 & 0.984 & $<0.01$ & $<0.01$ & $78.03 / 21.98$ \\
\hline 3 & 22 & -4203.76 & 8451.51 & 8536.39 & 8466.59 & 0.952 & $<0.01$ & $<0.01$ & $61.29 / 22.63 / 16.08$ \\
\hline 4 & 28 & -3992.92 & 8041.83 & 8149.85 & 8061.02 & 0.935 & 0.0665 & $<0.01$ & $43.5 / 32.31 / 11.28 / 12.91$ \\
\hline 5 & 34 & -3877.53 & 7823.07 & 7954.23 & 7846.37 & 0.932 & 0.4579 & $<0.01$ & $24.86 / 38.12 / 15.70 / 9.20 / 12.14$ \\
\hline
\end{tabular}

Note. The values in the LMR and BLRT columns are the $p$ values related to LMR and BLRT in comparing fit between models. $m=$ the number of free parameters;AIC = Akaike information criterion; BIC = Bayesian information criterion; $\mathrm{aBIC}=$ adjusted Bayesian information criterion; $\mathrm{LMR}=\mathrm{Lo}-\mathrm{Mendell}-$ Rubin;BLRT = Bootstrapped Likelihood Ratio Tests;

As shown in Table 4, the probabilities of Profiles 1, 2 and 3 of the three-profile model were $98 \%, 97 \%$ and $99.9 \%$, respectively. These indices suggested a good discriminability and reliable classification of the three-profile model.

Table 4 Average latent profile probabilities for most likely profile membership (row) by latent profile (column)

\begin{tabular}{|llll|}
\hline Profile & $1(n=217)$ & $2(n=77)$ & $3(n=56)$ \\
1 & 0.98 & 0.02 & 0.000 \\
2 & 0.03 & 0.97 & 0.004 \\
3 & 0.00 & 0.001 & 0.999 \\
\hline
\end{tabular}

Note. The columns refer to latent class, and the rows refer to most likely profile membership.

The scores of the three potential categories of mental health of empty nest elderly are as follows(Figure1, Table5): Profile 1 was the group with low ego, emotion, adaptive capacity, interpersonal communication, and cognitive function. The participants who possessed this profile comprised $61.3 \%$ of the sample. All the dimensions are at a lower level. We named this profile "low mental health." Profile 2 was the group with a moderate level of ego, emotion, adaptive capacity, interpersonal communication, and cognitive function. We named this profile "moderate mental health" . This group comprised $22.6 \%$ of the sample. Profile 3 was the group with high level of ego, emotion, adaptive capacity, interpersonal communication, and cognitive function.We named this profile"high mental health," and it comprised $16.1 \%$ of the sample.

Table 5 Mean, standard deviation, and normalized score for mental health dimensions of the three profiles

\begin{tabular}{|lllll|}
\hline Dimension & Class1 & Class2 & Class3 & Normalized score \\
\hline Ego & $18.47 \pm 3.61$ & $23.74 \pm 6.44$ & $32.50 \pm 2.68$ & $35.42 \pm 5.70$ \\
\hline Emotion & $32.66 \pm 3.42$ & $38.10 \pm 6.49$ & $46.41 \pm 3.10$ & $45.52 \pm 8.69$ \\
\hline Adaptive capacity & $43.68 \pm 4.24$ & $49.28 \pm 5.59$ & $57.85 \pm 3.64$ & $56.01 \pm 9.49$ \\
\hline Interpersonal communication & $16.672 \pm 3.37$ & $22.11 \pm 6.65$ & $30.98 \pm 2.87$ & $30.27 \pm 6.16$ \\
\hline Cognitive function & $17.91 \pm 3.68$ & $22.76 \pm 4.44)$ & $29.88 \pm 3.00$ & $26.36 \pm 5.46$ \\
\hline
\end{tabular}

Age, gender, marital status, degree of education, and household monthly income effect on the best-fitting latent profile of mental health's five dimensions

Gender, age, marital status, degree of education, and household monthly income were the independent variables; three potential categories of mental health were the dependent variable; and multinomial logistical regression was used to verify the significant factors that predicted potential categories of mental health, and take the Class1 as the reference group. The results of the three-step approach showed that when compared to the reference group, age, degree of education and marital status help to predict the potential categories of mental health, while other variables have no significant effect on the potential categories of mental health among empty-nest older adults, as shown in Table 6.

Table 6 Multinomial logistical regression results of demographic data on the three-profile model 


\begin{tabular}{|llllllllll|}
\hline Variable & \multicolumn{3}{c}{ Class2 } & \multicolumn{5}{c|}{ Class3 } \\
\hline Gender & $\beta$ & SE & $\beta /$ SE & $P$ & $\beta$ & SE & $\beta /$ SE & $P$ \\
\hline Age & -0.68 & 0.35 & -1.92 & 0.06 & -0.81 & 0.50 & -1.62 & 0.11 \\
\hline Degree of education & -0.08 & 0.03 & -2.97 & 0.00 & -0.13 & 0.05 & -2.68 & 0.01 \\
\hline Marital status & 1.26 & 0.32 & 3.99 & 0.00 & 2.57 & 0.52 & 4.99 & 0.00 \\
\hline household monthly income & 0.35 & 0.32 & 1.10 & 0.27 & 0.07 & 0.48 & 0.14 & 0.88 \\
\hline
\end{tabular}

\section{Comparing quality of life of mental health profiles}

To examine the outcomes of the three profiles, we compared the differences between quality of life and the related dimensions of each profile on the SF-36.

Table 7 presents the comparison of SF-36 outcome means among the three profiles

\begin{tabular}{|c|c|c|c|c|c|c|c|c|c|}
\hline \multirow{3}{*}{ M } & \multirow[b]{2}{*}{ Variable } & \multicolumn{2}{|c|}{ low mental health } & moderately mental health & \multicolumn{5}{|c|}{ high mental health } \\
\hline & & & & & & $\mathrm{F}$ & пр2 & & \\
\hline & SD & $M$ & SD & $M$ & SD & & & & \\
\hline RP & 62.95 & 5.26 & 70.53 & 4.98 & 75.91 & 6.72 & $149.72^{\star \star}$ & 0.46 & $\mathrm{C} 1 \otimes \mathrm{C} 2 \otimes \mathrm{C} 3$ \\
\hline $\mathrm{PF}$ & 53.12 & 2.97 & 58.10 & 2.74 & 64.64 & 5.04 & $283.55^{\star \star}$ & 0.62 & $\mathrm{C} 1 \otimes \mathrm{C} 2 \otimes \mathrm{C} 3$ \\
\hline BP & 73.58 & 13.18 & 69.27 & 11.66 & 62.57 & 16.50 & $15.58^{\star \star}$ & 0.08 & $\mathrm{C} 1 \rrbracket \mathrm{C} 2 \rrbracket \mathrm{C} 3$ \\
\hline $\mathrm{GH}$ & 54.65 & 3.78 & 60.40 & 4.84 & 66.54 & 6.48 & $168.06^{\star *}$ & 0.49 & $\mathrm{C} 1 \varangle \mathrm{C} 2 \varangle \mathrm{C} 3$ \\
\hline VT & 59.58 & 12.02 & 69.29 & 10.60 & 76.68 & 11.12 & $57.12^{* *}$ & 0.25 & $\mathrm{C} 1 \otimes \mathrm{C} 2 \bowtie \mathrm{C} 3$ \\
\hline $\mathrm{SF}$ & 61.34 & 9.70 & 67.56 & 10.08 & 74.25 & 10.48 & $41.70^{\star \star}$ & 0.19 & $\mathrm{C} 1 \llbracket \mathrm{C} 2 \llbracket \mathrm{C} 3$ \\
\hline RE & 64.81 & 4.99 & 72.47 & 6.52 & 77.86 & 8.96 & $120.63^{\star *}$ & 0.41 & $\mathrm{C} 1 \otimes \mathrm{C} 2 \llbracket \mathrm{C} 3$ \\
\hline $\mathrm{MH}$ & 58.16 & 6.86 & 65.97 & 6.31 & 72.57 & 6.50 & $119.26^{* *}$ & 0.41 & $\mathrm{C} 1 \otimes \mathrm{C} 2 \otimes \mathrm{C} 3$ \\
\hline
\end{tabular}

${ }^{*} \mathrm{p}<0.05,{ }^{* *} \mathrm{p}<0.01$

PF:Pysical functioning,RP: role-physical; BP:bodily pain; GH:general health;VT: vitality ;SF: social functioning; RE:role-emotional;mental health (MH);

\section{Correlation Analyses Between The Variables}

As presented in Table 2, total score of mental health of the elderly was positively related with education level, household monthly income, role-physical, pysical functioning, general health, vitality, social functioning, role-emotional, mental health, total score of quality of life $\left(r=0.618^{\star \star}, 0.407^{\star \star}, 0.711^{\star \star}, 0.869^{\star \star}, 0.681^{\star \star}\right.$, $0.554^{\star *}, 0.470^{\star *}, 649^{\star \star}, 0.677^{\star *}$ and $0.701^{\star *}$, respectively). The total score of mental health of the elderly was negatively correlated with gender, age, marital status, bodily pain $\left(r=-0.108^{*},-0.395^{\star *},-0.131^{\star}\right.$ and $-0.303^{\star *}$, respectively). 
Table 2

Correlation analysis among variables

\begin{tabular}{|c|c|c|c|c|c|c|c|c|c|c|c|c|}
\hline & 1 & 2 & 3 & 4 & 5 & 6 & 7 & 8 & 9 & 10 & 11 & 12 \\
\hline 1.gender & 1.000 & .002 & $-.169^{\star *}$ & -.013 & -.062 & $-.108^{\star}$ & .051 & -.056 & .043 & -.029 & $.199^{\star}$ & $.206^{\star *}$ \\
\hline 2.age & .002 & 1.000 & $-.431^{\star *}$ & $-.288^{\star \star}$ & .019 & $-.395^{\star *}$ & $-.372^{\star \star}$ & $-.298^{\star \star}$ & $.144^{\star \star}$ & $-.332^{\star \star}$ & $-.276^{\star \star}$ & $-.261^{\star \star}$ \\
\hline 3.Eu & $-.169^{\star *}$ & $-.431^{\star \star}$ & 1.000 & $.576^{\star *}$ & -.033 & $.618^{\star *}$ & $.575^{\star *}$ & $.551^{\star *}$ & $-.314^{\star \star}$ & $.562^{\star *}$ & $.408^{\star *}$ & $.355^{\star \star}$ \\
\hline 4. Hmi & -.013 & $-.288^{\star \star}$ & $.576^{\star \star}$ & 1.000 & .062 & $.407^{\star \star}$ & $.400^{\star \star}$ & $.409^{\star \star}$ & $-.196^{\star \star}$ & $.373^{\star \star}$ & $.311^{\star *}$ & $.264^{\star \star}$ \\
\hline 5.Mar & -.062 & .019 & -.033 & .062 & 1.000 & $-.131^{\star}$ & $-.110^{\star}$ & $-.130^{\star}$ & .016 & -.071 & $-.126^{*}$ & -.097 \\
\hline 6.EMH & $-.108^{\star}$ & $-.395^{\star \star}$ & $.618^{\star \star}$ & $.407^{\star \star}$ & $-.131^{*}$ & 1.000 & $.711^{\star \star}$ & $.869^{\star \star}$ & $-.303^{\star \star}$ & $.681^{\star \star}$ & $.554^{\star \star}$ & $.470^{\star \star}$ \\
\hline 7.RP & .051 & $-.372^{\star \star}$ & $.575^{\star \star}$ & $.400^{\star *}$ & $-.110^{*}$ & $.711^{\star *}$ & 1.000 & $.638^{\star *}$ & $-.382^{\star \star}$ & $.728^{\star \star}$ & $.826^{\star \star}$ & $.730^{\star \star}$ \\
\hline 8.PF & -.056 & $-.298^{\star \star}$ & $.551^{\star \star}$ & $.409^{\star \star}$ & $-.130^{\star}$ & $.869^{\star \star}$ & $.638^{\star \star}$ & 1.000 & $-.328^{\star \star}$ & $.615^{\star \star}$ & $.509^{\star \star}$ & $.417^{\star \star}$ \\
\hline 9.BP & .043 & $.144^{\star *}$ & $-.314^{\star *}$ & $-.196^{\star \star}$ & .016 & $-.303^{\star *}$ & $-.382^{\star \star}$ & $-.328^{\star \star}$ & 1.000 & $-.341^{\star \star}$ & $-.285^{\star \star}$ & $-.257^{\star \star}$ \\
\hline 10.GH & -.029 & $-.332^{\star \star}$ & $.562^{\star \star}$ & $.373^{\star \star}$ & -.071 & $.681^{\star *}$ & $.728^{\star \star}$ & $.615^{\star *}$ & $-.341^{\star \star}$ & 1.000 & $.483^{\star *}$ & $.390^{\star *}$ \\
\hline 11.VT & $.199^{\star \star}$ & $-.276^{\star \star}$ & $.408^{\star \star}$ & $.311^{\star *}$ & $-.126^{*}$ & $.554^{\star \star}$ & $.826^{\star *}$ & $.509^{\star \star}$ & $-.285^{\star \star}$ & $.483^{\star \star}$ & 1.000 & $.800^{\star \star}$ \\
\hline 12.SF & $.206^{\star *}$ & $-.261^{\star \star}$ & $.355^{\star *}$ & $.264^{\star \star}$ & -.097 & $.470^{\star *}$ & $.730^{\star *}$ & $.417^{\star *}$ & $-.257^{\star \star}$ & $.390^{\star *}$ & $.800^{\star *}$ & 1.000 \\
\hline 13.RE & -.013 & $-.319^{\star \star}$ & $.523^{\star \star}$ & $.343^{\star \star}$ & -.076 & $.649^{\star \star}$ & $.720^{\star \star}$ & $.584^{\star \star}$ & $-.343^{\star \star}$ & $.976^{\star \star}$ & $.469^{\star *}$ & $.376^{\star \star}$ \\
\hline 14.MH & $.129^{\star}$ & $-.336^{\star \star}$ & $.509^{\star \star}$ & $.368^{\star *}$ & $-.124^{\star}$ & $.677^{\star *}$ & $.925^{\star *}$ & $.608^{\star *}$ & $-.325^{\star \star}$ & $.581^{\star *}$ & $.933^{* *}$ & $.848^{\star *}$ \\
\hline 15.QOL & $.143^{\star \star}$ & $-.354^{\star \star}$ & $.499^{\star \star}$ & $.355^{\star \star}$ & $-.135^{*}$ & $.701^{\star *}$ & $.891^{\star *}$ & $.616^{\star *}$ & $-.110^{\star}$ & $.687^{\star \star}$ & $.886^{\star \star}$ & $.817^{\star \star}$ \\
\hline
\end{tabular}

Edu: education level; Hmi: Household monthly income;Mar:Marital status; EMH:Elderly Mental Health, PF:Pysical functioning,RP: role-physical; BP:bodily pain social functioning; RE:role-emotional;mental health (MH);QOL:Total score of quality of life

${ }^{*} \mathrm{p}<0.05,{ }^{* *} \mathrm{p}<0.01$

\section{Discussion}

The purpose of this article was to identify latent profiles of empty-nest elderly's mental health and then to examine how these different profiles were associated with empty-nesters' quality of life. In this study, we used latent profile analysis (LPA) to detect latent classes of mental health among empty-nest elderly. This classification method was objective which used five factors of the mental health, including ego, emotion, adaptive capacity, interpersonal communication, and cognitive function. This approach resulted in division of the data collected from 350 empty-nest elderly adults into three distinct profiles. The three-cluster model proved to be the best solution based on the model accuracy indices and with regard to reflections on the findings. A total of 217 cases experienced poor mental health, accounting for $61.29 \%$; 77 experienced moderate level of mental health, accounting for $22.63 \%$; 56 cases experienced a higher level of mental health, accounting for $16.08 \%$.

Empty nesters with low mental health (Profile 1) represented the largest group (61.3\% of the total sample), and they reflected just below-average scores on all five components, were characterized by low level of ego, disharmonious interpersonal communication and poor cognitive function, the main manifestations are emotional instability, poor adaptability, difficult to cope with stress events, weak frustration tolerance and resilience, inappropriate self-evaluation, easy to underestimate themselves, and poor interpersonal skills. The result shows that low mental health group is psychologically fragile, has certain risks in the face of stress or setbacks, and may develop into serious psychological problems. Therefore, this group is the object that needs special attention in practical work, and early targeted intervention is helpful to improve the mental health level of the empty-nest elderly adults. Community workers should give them mental health education in time, prevent psychological obstacles and, if necessary, carry out psychological counseling to improve their mental health status[26].

The group situated between the low and high mental health empty-nest elderly (Profile 2) represented $22.6 \%$ of the total sample. They demonstrated a moderate level of mental health, however, the score of interpersonal communication ability dimension is low[27]. The interpersonal relationship of the emptynest elderly adults is mainly reflected in the relationship between husband and wife, with their children and friends. Due to the narrowing of the scope of social communication, elderly parents turn their attention to their children, but empty-nest elderly's families weaken the emotional relationship between elderly parents and their children to a certain extent, because lack the companionship of their children, empty-nest elderly are prone to negative emotions such as anxiety, pessimism and loss, which are strong or lasting repeated experiences, that is, they become a kind of long-term spiritual stimulation. It is bound to cause varying degrees of harm to the mental health of the empty nest elderly. And with the increase of age, the lack of physical strength and the inconvenience of movement will seriously affect the interpersonal communication of the elderly[28]. Therefore, the establishment of good interpersonal relationship is of great significance to the maintenance of mental health of the general empty nest elderly. The community should actively organize various types of activities for the elderly and distribute small gifts to provide the enthusiasm of the elderly to participate in activities and expand the scope of 
interpersonal communication. Empty-nest elderly are encouraged to increase their social participation through the Internet or telephone, so as to enrich their inner life and improve their mental health.

Empty-nest elderly with high mental health (Profile 3) represented nearly $16.1 \%$ of the total sample, but the score of cognitive function dimension was lower than that of the norm, which is consistent with a previous study[29].Cognitive function is not only a sensitive index of normal aging of psychological function, but also a measure of pathological aging (such as Alzheimer's disease). Cognitive aging has a negative impact on the quality of life and mental health of empty-nest elderly. According to the emotional ABC theory[30],if the belief and cognition of the empty nest elderly can be corrected, the negative life experience will be weakened, which shows that the cognitive correction and self-regulation of the empty nest elderly is very important. Empty-nest elderly actively participate in various sports and intellectual activities and maintain an optimistic and cheerful state of mind, which plays a promoting role in delaying the decline of cognitive function[31]. Therefore, delaying cognitive aging will be an important aspect of mental health maintenance for empty-nest elderly. Regarding quality of life, our profile-based findings mirror the results of studies using other approaches(i.e. variable-centered approach, which dominated empirical nursing care research) that have found that empty nesters with higher levels of mental health tend to enhance their quality of life.

This study further discussed the differences of mental health potential categories of empty nest elderly in related demographic variables. The distribution of mental health subtypes of empty-nest elderly has no significant difference in gender and family monthly income, but has significant difference in age, education level and marital status. With advanced age and low level of education, single empty nesters are more likely to be distributed in "low mental health" groups. With the growth of age, the physical health and mental health of the elderly have appeared a series of problems, and the special group of empty-nest elderly due to aging and family factors, their mental health status is more worrying. Previous studies have shown that the negative detection rates of depression and anxiety among community empty nesters are $23.2 \%[32]$ and $24.7 \%$ [33], respectively. Poor mental health significantly reduces the quality of life of empty-nest elderly, and is also closely related to senile dementia, chronic disease and death[34]. The empty-nest elderly with a higher level of education have more ways of entertainment and a richer life; at the same time, they can master some psychological common sense and adjust their bad mood in time, so as to effectively dispose of loneliness and loneliness in life[35]. Marital status has a significant effect on the subgroup classification of mental health of the empty-nest elderly. the mental health level of the single empty-nest elderly is lower than that of the married empty-nest elderly, which may be related to their living alone and lack of family warmth[36]. A sound family structure can give adequate support to the elderly at the material and spiritual levels. At the same time, full communication and emotional sharing with spouses also reduce the loneliness of the elderly to a certain extent, which is conducive to improving the mental health of the elderly. Age, education level and marital status are not only important factors affecting mental health, but also important factors of potential category differentiation of empty nest elderly.

\section{Limitations}

Although the current study yielded important and practical findings on the targeted intervention and guidance, several limitations should be noted. First of all, cross-sectional data limits the interpretation of the findings as causal claims cannot be made. Where possible, future research should aim for longitudinal data collection to better understand the process of mental health development among empty-nest olderly. Second, the sample population of empty-nest elderly included only a few selected communities of Chifeng City in Inner Mongolia. Thus, these findings may not be representative of the older adults in other geographic areas within the province.

\section{Conclusions}

The results of this study suggest that there are three profiles of empty-nest elderly in regard to mental health: low mental health, moderate mental health, and high mental health. In addition, our study has shown that empty-nest elderly with low mental health, moderate mental health, and high mental health have significant differences in their quality of life. As such, this study provides compelling evidence that focusing on the specific dimensions of mental health, and the patterns in which they actually occur in empty-nest elderly people. Therefore, different interventions for different profiles of empty-nest elderly are beneficial to raise all the empty-nest elderly's mental health and quality of life.These results provide a new and expanded view of empty-nest elderly's mental health,which can help China's leaders better target investments and create policies aimed at improving the mental health and quality of life among empty-nest elderly in China.

\section{Abbreviations}

LPA:Iatent profile analysis; QoL:Quality of life; AIC: Akaike information criterion, BIC:Bayesian information criterion, aBIC: adjusted BIC, LMR:Lo-Mendell-Rubin, BLRT:Bootstrapped Likelihood Ratio Test.

\section{Declarations}

\section{Acknowledgements}

The authors would like to thank all the participants who voluntarily participated in this study and research assistants who performed the data collection.

\section{Authors' contributions}

Hong Su made the contributions on study design, data collection, analysis, interpretation, and wrote the paper. Yuqiu Zhou provided guidance in the study design, and is the corresponding author. Yunjiang Cai made contributions on investigation execution and data analysis. Yun Wang assisted with the data interpretation. All authors have read and approved the final version of the manuscript.

\section{Funding}


This research was supported by“ The Fundamental Research Funds for the Provincial Universities,funds number"2018wld-01" and College of nursing cultivation fund, fund number"HLPY1803".,

\section{Availability of data and materials}

The datasets used and/or analysed during the current study are available from the corresponding author on reasonable request.

\section{Ethics approval and consent to participate}

All patients provided their informed written consent. The study was approved by the Ethics Committee of Harbin Medical University and was conducted in accordance with the principles of the Declaration of Helsinki.

\section{Consent for publication}

Not applicable.

\section{Competing interests}

The authors declare that they have no competing interests

\section{Author details}

${ }^{1}$ Department of Nursing, Daqing Campus, University of Harbin Medical,39 Shinyo Road, Daqing District, Heilongjiang 163319, China

\section{References}

1. National bureau of statistics of the People's Republic of China. China Statistical Yearbook 2019 (in Chinese). Beijing: China Statistic Press.

2. Chen, Zhengying. Chu Ting. Investigation on depression of empty nest elderly in ethnic areas and its influencing factors. Chin J Gerontol. 2012;32(1):130-1.

3. Wei M, Xinli M. Huang Ziqi. Study on the relationship between Family Care and alexithymia of empty Nest elderly in Community. Chinese general practice. 2020;18(4):612-4.

4. Chang Y, Guo X, Guo L, et al. Comprehensive comparison between empty nest and non-empty nest elderly: a cross-sectional study among rural populations in northeast China. Int J Environ Res Public Health. 2016;13:857.

5. Huang YJ, Zhao YY, Wu YL,et al. SCL-90 test result on the empty nest elderly in China: a meta-analysis. Chin J Dis Control Prev. 2016;20(1):74-8.

6. Gao YL, Wei YB, Shen YD, Tang YY, Yang JR. China's empty nest elderly need better care. J Am Geriatr Soc. 2014;62:1821-2.

7. Su H, Wang LN, Zhou YQ, Cao JY. A comparative study on the relationship between loneliness and quality of life of urban and rural empty-nest elderly in Inner Mongolia Autonomous region. Chinese Journal of practical Nursing. 2015;31(12):926-30.

8. Zheng DW, Liu XQ. Mental health status of empty nest elderly and its influencing factors. Chin J Gerontol. 2017;37(20):5174-5.

9. Qiu HZ. The principle and technology of potential category model [M]. Beijing: Peking University Press; 2008.

10. Magidson J, Vermunt JK. Latent class models for clustering: a comparison with K-means. Canad J Market Res. 2002;20(1):37-44.

11. Marsh HW, Lüdtke O, Trautwein U,et al. Classical latent profile analysis of academic self-concept dimensions: Synergy of person-and variablecentered approaches to theoretical models of self-concept. Structural Equation Modeling: A Multidisciplinary Journal. 2009;16:191-225.

12. Browning C. Excerpts of 2007 Beijing international academic summit of general practice-the role of general practitioners in promoting health ageing. Chinese General Practice. 2007;10:1131-2.

13. Wiggins RD, Higgs PF, Hyde M, et al. Quality of life in the third age:key predictors of the CASP-19 measure. Ageing\&Society. 2004;24(5):693-708.

14. Guo YQ, Zheng X, Pan XJ,et al. Mental health status and influencing factors of empty nest elderly. Chin J Gerontol. 2017;37(4):967-70.

15. Gu HX. Quality of life and psychological and social support of empty nest elderly.

16. Chinese Journal of Health Psychology,Journal of Health Psychology,2015; 23 (8): 1218-1222.

17. Jing X, Chen J, Dong Y, et al. Related factors of quality of life of type 2 diabetes patients: A systematic review and meta-analysis. Health Quality of Life Outcomes. 2018;16(1):189.

18. Cao WJ, Wu JJ, Zeng NJ,et al. Study on the relationship between anxiety, depression and quality of life of chronic empty-nest elderly in poverty-stricken counties of Hunan province. Nurs Res. 2020;34(5):784-8.

19. Wu ZY, Xu SL, Li J. The development of elderly mental health questionaire. J Clin Psychol. 2002;10(1):1-3.

20. Lam CLK, Tse EYY, Gandek B, et al. The SF-36 summary scales were valid, reliable, and equivalent in a Chinese population. J Clin Epidemiol. 2005;58:815-22.

21. Ware JE, Gandek B. Overview of the SF-36 health survey and the international quality of life assessment (IQOLA) project. J Clin Epidemiol. 1998;51:90312.

22. Li D, Xu T, Wu DW,et al. Reliability and validity of SF-36 scale in elderly general population. Chinese Journal of Rehabilitation Medicine, 2004 ; (7): $35-37$.

23. Carragher N, Adamson G, Bunting B, et al. Subtypes of depression in a nationally representative sample. J Affect Disord. 2009;113(1):88-99.

24. Lo YT, Mendell NR, Rubin DB. Testing the number of components in a normal mixture. Biometrika. 2001;88:767-78.

25. Asparouhov T, Muthén B. Auxiliary variables in mixture modeling: three-step approaches using Mplus. Struct Eq Model. $2014 ; 21(3): 329-41$.

26. Kongsted A, Nielsen AM. Latent Class Analysis in health research. J Physiother. 2017;63(1):55-8. 
27. Gu RM, Song HX, Li Jian Z. Investigation and correlation study on social support and mental health status of the elderly in community. Chinese General Medicine. 2019;22(5):570-4.

28. Zhang LH, Ke Y, Wu ZX. An Analysis of interpersonal Communication and Social support of empty Nest elderly. Journal of Science Technology Entrepreneurship. 2017;30(2):111-2.

29. Wang DH, Laidlaw K, Power MJ,et al. Older people's belief of filial piety in China:Expectation and non-expectation.Clinical Geronologist.2009; 33(1):21-38. 30. Ge Qingjun. Study on mental health status and influencing factors of empty nest elderly. Monthly Journal of Psychology. 2019;14(13):53.

31. Xu, Bing. Tang Bolin.The enlightenment of Ellis ABC theory to the construction of college students' individual emotion regulation system. Agricultural Education Research. 2009;(1):22-24.

32. Li DM, Chen TY. Cognitive aging and mental health of the elderly. Development of Psychological Science. 2006;14(4):560-3.

33. Ding Y, Yan CR, Ma XL, et al. Analysis of anxiety and depression status and influencing factors of empty nest elderly in community. Anhui Medicine. 2019;40(8):947-50.

34. Yang WM, Fu HP, Liu SH, et al. Effect of community intervention on anxiety and depression of empty nest elderly. Chin J Gerontol. 2012;32(4):813-4.

35. Hu J, Liu XJ, Dai XY, et al. Relationship between social support, coping style, self-efficacy and depression of empty-nest elderly in Wuhan community. Chin J Gerontol. 2018;38(6):1508-11.

36. Guo TX, Sun GQ. Research progress on mental health status and related factors of empty nest elderly. Electronic Journal of Cardiovascular Diseases with Integrated traditional Chinese Western Medicine. 2018;6(36):11-4.

37. Hao Y, Tao L, Li LL, et al. Analysis of mental health status and influencing factors of empty nest elderly in Xi'an. Chinese Journal of Social Medicine. 2018;35(3):237-9.

\section{Figures}

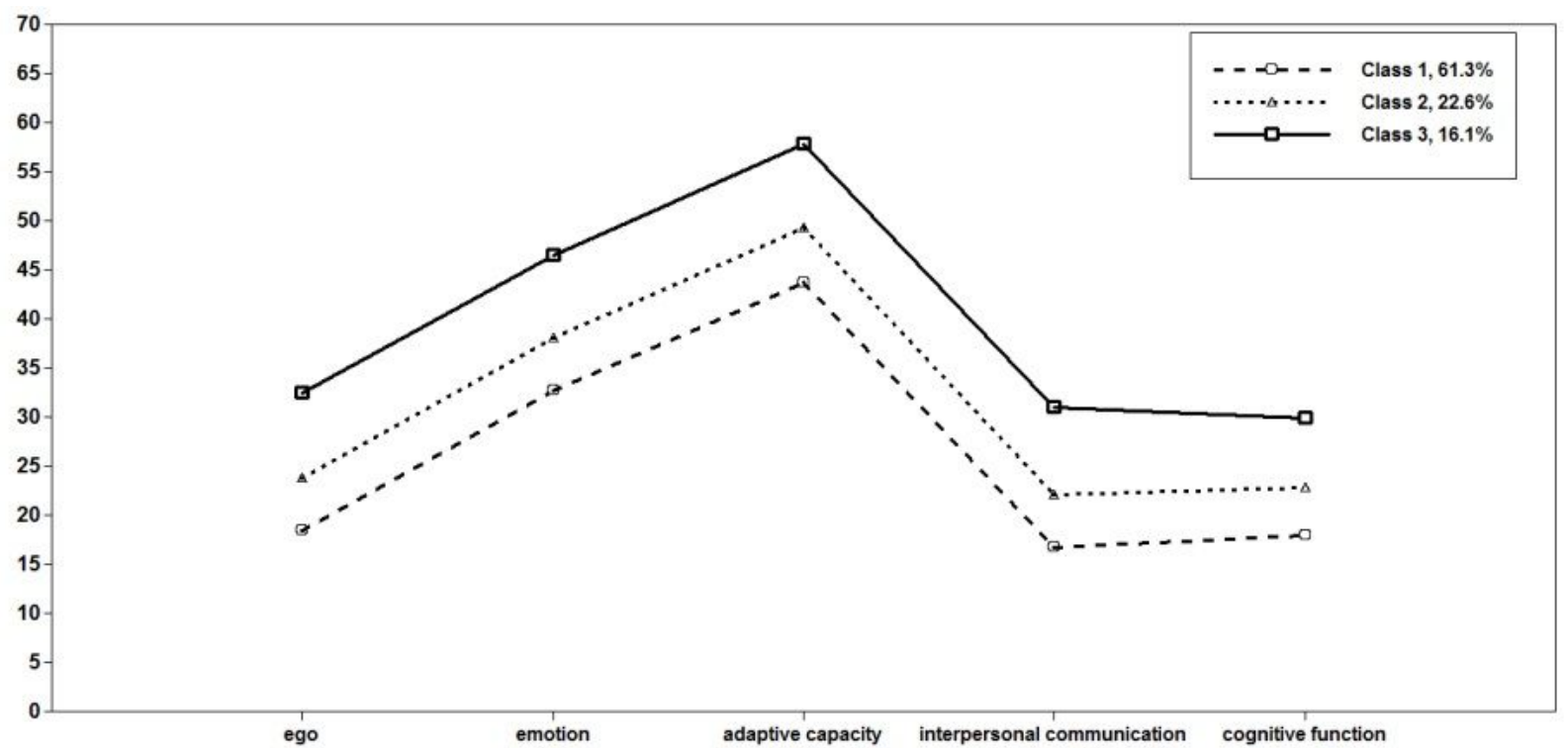

Figure 1

Mean scores on ego, emotion, adaptive capacity, interpersonal communication, and cognitive function of mental health in Chinese empty-nest older adults 